\title{
The effect of anisotropic gas pressure on Alfvén-wave transmission and test-particle acceleration at parallel shock waves
}

\author{
R. Vainio ${ }^{1,2, \star}$ and R. Schlickeiser ${ }^{2}$ \\ 1 Space Research Laboratory, Department of Physics, 20014 Turku University, Finland \\ 2 Insitut für Theoretische Physik, Lehrstuhl IV: Weltraum- und Astrophysik, Ruhr-Universität Bochum, \\ 44780 Bochum, Germany
}

Received 16 February 2001 / Accepted 27 August 2001

\begin{abstract}
Alfvén-wave transmission through a parallel shock in case of an anisotropic gas pressure $\left(P_{\|} \neq P_{\perp}\right)$ is considered from the point of view of test particle acceleration in such shock waves. Treating the shock's gas compression ratio and the firehose factor, $\phi=1-4 \pi\left(P_{\|}-P_{\perp}\right) / B^{2}$, on both sides of the shock as given parameters, we calculate the wave-transmission coefficients for circularly-polarized Alfvén waves of finite amplitude. We also give an equation for the shock's gas compression ratio including the effects of pressure anisotropy and waves. We study wave transmission and test-particle acceleration in a model, where the downstream plasma is isotropic and the upstream plasma remains stable against the firehose instability $(\phi>0)$. The results can be arranged in a following manner with respect to the upstream parallel plasma beta, $\beta_{\|} \equiv 8 \pi P_{\|} / B_{0}^{2}$ (with $B_{0}$ being the ordered magnetic field): (i) for shocks with $\beta_{\|} \ll 2$ the results for particle acceleration are only quantitatively different for anisotropic upstream plasma; (ii) for $\beta_{\|} \sim 2$, large anisotropies alter the wave-transmission and particle acceleration picture completely relative to the isotropic case yielding much harder energy spectra with spectral indices $1<\Gamma<2$ for all shocks with $1<r<4$; (iii) for very hot and firehose-stable plasmas, the qualitative changes in particle acceleration due to pressure anisotropies are limited to the weakest shocks and, thus, should be analyzed in a non-linear manner to confirm the results.
\end{abstract}

Key words. acceleration of particles - shock waves - turbulence

\section{Introduction}

Diffusive shock acceleration (Axford et al. 1977; Krymsky 1977; Bell 1978; Blandford \& Ostriker 1978) is a universal mechanism for the acceleration of non-thermal particle populations (Drury 1983; Blandford \& Eichler 1987). Since energetic particles can be confined near the shock by plasma turbulence, they are accelerated due to two effects: (i) first-order Fermi acceleration due to multiple crossings of the shock compression front and (ii) stochastic second-order Fermi acceleration in the turbulent fields near the shock. Assuming that the turbulence consists of low-frequency waves on both sides of the shock enables one to investigate the relative importance of these two mechanisms. In this picture, the presence of the second-order Fermi effect requires waves propagating in both directions relative to the mean magnetic field. This condition is

Send offprint requests to: R. Vainio, e-mail: ravainio@utu.fi

* Permanent address: Turku University. always satisfied in the shock's downstream region since an upstream Alfvén wave, when transmitted through a fastmode shock, converts to two downstream waves propagating in both directions relative to the field (McKenzie \& Westphal 1969). However, a consideration of the acceleration time scales of the two mechanisms shows that (i) is much faster than (ii), and should dominate acceleration under the assumption that the upstream waves are self-generated by the cosmic rays streaming against the plasma flow and, therefore, propagating in the backward direction relative to the flow (Vainio \& Schlickeiser 1998, 1999).

When the second-order Fermi acceleration is neglected one obtains the canonical power-law (over momentum) test-particle energy spectrum,

$\mathrm{d} J / \mathrm{d} E \propto p^{-\Gamma}$

at the shock. Here, $\Gamma=\left(r_{k}+2\right) /\left(r_{k}-1\right)$ and $r_{k}=V_{n, 1} / V_{n, 2}$ is the scattering-center compression ratio at the shock, 
$V_{n, 1[2]}$ is the normal component of the mean velocity of the upstream [downstream] scattering centers as measured in the frame of the shock. In general, the scattering center compression ratio is not the same as the gas compression ratio $r=u_{n, 1} / u_{n, 2}$ given by the ratio of normal plasma flow speeds, $u_{n, 1[2]}$. In particular, scattering-center compression is not limited by the limiting value of $r<\left(\gamma_{\mathrm{g}}+1\right) /\left(\gamma_{\mathrm{g}}-1\right)$, where $\gamma_{\mathrm{g}}$ is the polytropic index of the gas equaling $5 / 3$ for an isotropic, adiabatic, non-relativistic gas.

Vainio \& Schlickeiser (1998) studied the transmission of Alfvén waves through a parallel shock wave and calculated the resulting scattering-center compression ratio for a model neglecting the effect of waves on the shock's gas compression ratio. They showed that the scatteringcenter compression ratio tends to infinity at shocks with $r \rightarrow\left(\gamma_{\mathrm{g}}+1-\gamma_{\mathrm{g}} \beta\right) /\left(\gamma_{\mathrm{g}}-1\right)$, where $\beta \equiv 8 \pi P_{1} / B_{0}^{2}$ is the upstream plasma beta ${ }^{1}$, and $P_{1}$ and $B_{0}$ are the (isotropic) upstream gas pressure and the ordered magnetic field, respectively. At this limit, the Alfvénic Mach number of the shock, $M \equiv u_{1} / V_{\mathrm{A} 1} \rightarrow r^{1 / 2}$, and the downstream backward Alfvén waves become standing waves in the shock frame yielding formally infinite intensities for these waves. This singularity is not a physical one, and is removed by the inclusion of wave pressure and energy flux to the shock's Rankine-Hugoniot equations (Vainio \& Schlickeiser 1999). The scattering-center compression ratios remain high also in the self-consistent wave-transmission model which, therefore, is able to produce test-particle spectra that are harder than the limiting value of $\Gamma=2$ occurring for $r_{k}=r=4$ in the original magnetostatic formulation of diffusive test-particle acceleration at non-relativistic shocks.

Anisotropic gas pressure modifies the dispersion relation of the low-frequency (Alfvén) waves. As noted by Schlickeiser \& Vainio (1998), the important effects on particle acceleration in the model of Vainio \& Schlickeiser $(1998,1999)$ were the result of the inclusion of finite phase speeds of the waves into the theory. Furthermore, pressure anisotropies also modify the jump relations at the shock in a non-trivial way. We know from observations that the pressure in the solar wind is anisotropic (see, e.g., Marsch 1991 for a review). Particle reflection from the shock also adds its contribution to the pressure anisotropy, so the gas pressure just upstream a shock is probably anisotropic even if the far upstream conditions were isotropic.

The purpose of this paper is to investigate the influence of pressure anisotropies on the wave transmission problem, shock compression ratios, and the resulting test-particle acceleration at parallel shocks. Although we will discuss briefly the pressure anisotropies associated with nonthermal particles, it is beyond the scope of the present study to develop a theory with self-consistent determination of pressure anisotropies around the shock. Thus, the effects

\footnotetext{
1 Note that Vainio \& Schlickeiser (1998) defined plasma beta as the ratio of upstream sound speed to the upstream Alfvén speed, which equals $2 \beta / \gamma_{\mathrm{g}}$ or $6 \beta / 5$ for $\gamma_{\mathrm{g}}=5 / 3$ in this paper.
}

of anisotropy are studied in a simplified way treating the anisotropy of the gas on both sides of the shock as a known parameter, and constraining its values so that the plasma stays stable against the upstream firehose stability criterion. Similar ideas have been employed previously by, e.g., Lyu \& Kan (1986) in a study of plasma parameters at shocks measured in near-Earth space.

\section{The model}

\subsection{Shock equations}

In a plasma with a non-zero magnetic field, the motion of the particles along the magnetic field lines differs fundamentally from their motion perpendicular to the field lines. This points at the necessity of allowing different amounts of random energy for particle motions parallel and perpendicular to the field, respectively. One expects the distribution function to have an axial symmetry around the local magnetic field $\boldsymbol{B}$, if the Larmor radii of the particles are very small compared to the density scale in the perpendicular direction. Then the gas pressure is a tensor

$\mathrm{P}=P_{\perp} \mathrm{I}+\left(P_{\|}-P_{\perp}\right) \boldsymbol{B} \boldsymbol{B} / B^{2}$,

where $\mathbf{I}$ is the unit tensor. In a steady state, we can write all the macroscopic conservation laws in form (e.g., Boyd \& Sanderson 1969)

$\nabla \cdot \boldsymbol{\Phi}^{\alpha}=0$

where $\boldsymbol{\Phi}^{\alpha}$ is one of the fluxes: the mass flux,

$\boldsymbol{\Phi}^{m}=\rho \boldsymbol{u}$

the momentum flux,

$\boldsymbol{\Phi}^{p}=\rho \boldsymbol{u} \boldsymbol{u}+\mathrm{P}-\mathrm{T}$,

with

$\mathrm{T}=\boldsymbol{B} \boldsymbol{B} / 4 \pi-\left(B^{2} / 8 \pi\right) \mathbf{I}$

giving the electromagnetic stress tensor (neglecting small electric-field contributions); or energy flux

$\boldsymbol{\Phi}^{E}=\frac{c}{4 \pi} \boldsymbol{E} \times \boldsymbol{B}+\mathrm{P} \cdot \boldsymbol{u}+\frac{1}{2}\left(\operatorname{Tr} \mathrm{P}+\rho u^{2}\right) \boldsymbol{u}$,

which holds for a non-relativistic gas with zero heat flux. Here, $\operatorname{Tr} \mathrm{P}=2 P_{\perp}+P_{\|}$is the trace of the pressure tensor.

We consider the conservation laws across a planar shock discontinuity. In addition to the macroscopic conservation laws, we use Maxwell's equations $\nabla \cdot \boldsymbol{B}=0$ and $c^{-1} \partial \boldsymbol{B} / \partial t+\nabla \times \boldsymbol{E}=0$, and Ohm's law in form $\boldsymbol{E}=-\boldsymbol{u} \times \boldsymbol{B} / \mathrm{c}$ to arrive at jump conditions (see also Lyu \& Kan 1986)

$$
\begin{array}{r}
{\left[B_{n}\right]_{1}^{2}=0, \quad\left[\boldsymbol{B}_{t} u_{n}-B_{n} \boldsymbol{u}_{t}\right]_{1}^{2}=0} \\
{\left[\rho u_{n}\right]_{1}^{2}=0, \quad\left[\rho \boldsymbol{u}_{t} u_{n}-\phi \frac{B_{n} \boldsymbol{B}_{t}}{4 \pi}\right]_{1}^{2}=0} \\
{\left[\rho u_{n}^{2}+P_{\|}+(2 \phi-1) \frac{B_{t}^{2}}{8 \pi}\right]_{1}^{2}=0}
\end{array}
$$




$$
\begin{aligned}
& {\left[\left\{\frac{\rho}{2} u_{n}^{2}+\frac{5 P_{\|}}{2}+(\phi-1) \frac{B_{n}^{2}}{4 \pi}\right\} u_{n}\right.} \\
& \left.\quad+\left\{\frac{\rho}{2} u_{t}^{2}+(2 \phi-1) \frac{B_{t}^{2}}{4 \pi}\right\} u_{n}-\phi B_{n} \frac{\boldsymbol{u}_{t} \cdot \boldsymbol{B}_{t}}{4 \pi}\right]_{1}^{2}=0
\end{aligned}
$$

expressing the continuity of normal magnetic and tangential electric fields across the shock (Eq. (8)), and the conservation of mass and perpendicular momentum (Eq. (9)), parallel momentum (Eq. (10)), and energy (Eq. (11)). Here, subscripts $n$ and $t$ denote vector components normal and tangential to the planar shock and

$\phi \equiv 1-\frac{P_{\|}-P_{\perp}}{B^{2} / 4 \pi}$,

is the firehose factor specifying the pressure anisotropies. Regarding the upstream values for all variables as known input parameters and the downstream values as unknowns, we have altogether 8 equations and 9 unknowns. To close the set, we simply take the downstream pressure to be isotropic, which should be reasonable at least for shocks with a compression ratio differing considerably from unity; for such shocks the transition layer is likely to produce short-scale magnetic turbulence that can lead to the isotropization of the particles just downstream the shock.

\subsection{Alfvén-wave properties}

Pressure anisotropies also modify the dispersion relation of Alfvén waves, which at long-wave-length limit becomes (e.g., Stix 1962)

$\omega^{2}=\phi V_{\mathrm{A}}^{2} k^{2}$

for circularly polarized waves propagating parallel to the mean field. Here, the wave frequency $\omega$ and the wavenumber $k$ are measured in the plasma frame. For positive values of $\phi$, Eq. (13) describes waves propagating at phase speed $\pm \phi^{1 / 2} \boldsymbol{B}_{0}(4 \pi \rho)^{-1 / 2}$ parallel $(+)$ or anti-parallel $(-)$ to the mean magnetic field, $\boldsymbol{B}_{0}$. For negative values of $\phi$, Eq. (13) describes the firehose instability: the magnetic fluctuations become group-standing and grow exponentially at rate $V_{\mathrm{A}}|\phi|^{1 / 2}|k|$. From now on we take $\phi \geq 0$, although the results could be generalized also to the firehose-unstable case. Note that the firehose unstable case can only be realized for $P_{\|}>B^{2} / 4 \pi$, so restricting the analysis to the stable cases is natural for plasmas with low $\beta$.

Combining Ohm's and Faraday's laws gives the relation

$\delta \boldsymbol{u}^{ \pm}=\mp \phi^{1 / 2} \frac{\delta \boldsymbol{B}^{ \pm}}{(4 \pi \rho)^{1 / 2}}$

between the velocity and magnetic-field perturbations associated with Alfvén waves propagating parallel $(+)$ or anti-parallel $(-)$ to the mean magnetic field. We assume that the turbulent magnetic field can be decomposed to two wave fields propagating in opposite directions,

$\delta \boldsymbol{B}=\delta \boldsymbol{B}^{+}+\delta \boldsymbol{B}^{-}$, yielding the equation

$\delta \boldsymbol{u}=\phi^{1 / 2} \frac{\delta \boldsymbol{B}^{-}-\delta \boldsymbol{B}^{+}}{(4 \pi \rho)^{1 / 2}}$.

\section{Results and discussion}

\subsection{Alfvén-wave transmission and compression ratio}

If $M>\phi_{1}^{1 / 2}$ and $M / r^{1 / 2}>\phi_{2}^{1 / 2}$, the flow around the shock is entirely super-Alfvénic. Then all upstream Alfvén waves are convected through the shock to become downstream Alfvén waves. Some waves are transmitted retaining their propagation direction relative to the flow, but others get reflected, i.e., assume the other propagation direction. Alfvén-wave transmission through a parallel shock wave can be calculated by taking the tangential magneticfield and velocity components to be due to waves, $\boldsymbol{B}_{t}=$ $\delta \boldsymbol{B}$ and $\boldsymbol{u}_{t}=\delta \boldsymbol{u}$. We shall first investigate the transmission coefficients in a shock with a pre-described gas compression ratio. Thus, by using the first four jump conditions, Eqs. (8-9), together with Eqs. (15-16) and assuming a degenerate upstream cross helicity, $\delta \boldsymbol{B}_{1}=\delta \boldsymbol{B}_{1}^{ \pm}$, we find after an amount of straight-forward algebra

$T \equiv \frac{\delta B_{2}^{h}}{\delta B_{1}^{h}}=\frac{r^{1 / 2}\left(\phi_{2}^{1 / 2} r^{1 / 2}+\phi_{1}^{1 / 2}\right)}{2 \phi_{2}^{1 / 2}} \frac{M+h \phi_{1}^{1 / 2}}{M+h \phi_{2}^{1 / 2} r^{1 / 2}}$
$R \equiv \frac{\delta B_{2}^{-h}}{\delta B_{1}^{h}}=\frac{r^{1 / 2}\left(\phi_{2}^{1 / 2} r^{1 / 2}-\phi_{1}^{1 / 2}\right)}{2 \phi_{2}^{1 / 2}} \frac{M+h \phi_{1}^{1 / 2}}{M-h \phi_{2}^{1 / 2} r^{1 / 2}}$

where $h=+[-] 1$ denotes waves propagating parallel [antiparallel] to the plasma flow. These reduce to the results of Vainio \& Schlickeiser (1998) when $\phi_{1}=\phi_{2}=1$. The transmission problem was first treated by McKenzie \& Westphal (1969), but their results for anisotropic conditions are incorrect: they used an incorrect dispersion relation $\left(\omega / k= \pm \phi^{-1 / 2} V_{\mathrm{A}}\right)$ and neglected the additional $\phi$-factor from the transverse momentum equation (see Eq. (9)).

Let us, next, calculate the gas compression ratio for a shock propagating with Mach number $M$ into a gas with parallel plasma beta, $\beta_{\|} \equiv 8 \pi P_{\| 1} / B_{0}^{2}$ and firehose factor

$\phi_{1}=1-\frac{\beta_{\|}-\beta_{\perp}}{2(1+b)} \geq 0$,

with $\beta_{\perp} \equiv 8 \pi P_{\perp 1} / B_{0}^{2}$ and $b \equiv\left(\delta B / B_{0}\right)^{2}$ giving the perpendicular plasma beta and the squared ratio of the waveamplitude and ordered magnetic field $B_{0}$, respectively ${ }^{2}$. Eqs. (17-18) give

$\frac{B_{t 2}}{B_{t 1}}=T+R=r \frac{M^{2}-\phi_{1}}{M^{2}-\phi_{2} r}$,

which can be seen to hold for a general upstream cross helicity by transforming Eq. (9) into the de HoffmannTeller frame, where $\boldsymbol{u}_{t} B_{n}=u_{n} \boldsymbol{B}_{t}$ on both sides of the

\footnotetext{
${ }^{2}$ Note that this ratio was denoted $b^{2}$ in Vainio \& Schlickeiser (1999).
} 
shock. In this frame the energy equation can be written in a form independent of tangential velocities as

$$
\left[\left(\frac{\rho}{2} u_{n}^{2} \frac{B^{2}}{B_{n}^{2}}+\frac{5 P_{\|}}{2}+(\phi-1) \frac{B^{2}}{4 \pi}\right) u_{n}\right]_{1}^{2}=0
$$

with $B^{2}=B_{n}^{2}+B_{t}^{2}$. Because it does not involve tangential velocities, Eq. (21) holds in any shock frame. Using Eqs. (20-21) together with Eq. (10) gives two equations,

$$
\begin{aligned}
\frac{8 \pi P_{\| 2}}{B_{0}^{2}}= & \beta_{\|}+2 M^{2} \frac{r-1}{r} \\
& +b\left\{2 \phi_{1}-1-\left(2 \phi_{2}-1\right) r^{2} \frac{\left(M^{2}-\phi_{1}\right)^{2}}{\left(M^{2}-\phi_{2} r\right)^{2}}\right\}(22) \\
= & r \beta_{\|}+\frac{2}{5}\left\{M^{2} \frac{r^{2}-1}{r}+2\left[r\left(\phi_{1}-1\right)-\left(\phi_{2}-1\right)\right]\right. \\
& +b r\left[M^{2}+2\left(\phi_{1}-1\right)\right. \\
& \left.\left.-\left[M^{2}+2 r\left(\phi_{2}-1\right)\right] \frac{\left(M^{2}-\phi_{1}\right)^{2}}{\left(M^{2}-\phi_{2} r\right)^{2}}\right]\right\}
\end{aligned}
$$

for the downstream parallel pressure, where $B_{1 n, 2 n}=$ $B_{0}$ and $B_{1 t}^{2}=b B_{0}^{2}$ have been used. Multiplying both sides by $r\left(M^{2}-\phi_{2} r\right)^{2}$ produces a quartic equation for $r$, which reduces to a cubic given by Eq. (14) of Vainio \& Schlickeiser (1999) for $\phi_{1}=\phi_{2}=1$. The extra root appears in the present model, since $r=1$ is no longer a trivial solution, but represents a change in the pressure anisotropy accompanied by a change in the transverse magnetic field and parallel gas pressure.

One should also check for the physicality of the obtained shock solutions. We will not analyze this in detail, but note only that if the analysis of the entropy change across the shock is based on ideal gas law, we can write (Lyu \& Kan 1986)

$\Delta S \propto \ln \frac{P_{\perp 2}^{2} P_{\| 2} \rho_{1}^{5}}{P_{\perp 1}^{2} P_{\| 1} \rho_{2}^{5}}$.

Thus, for upstream $\beta_{\perp} \rightarrow 0$, the entropy change approaches infinity for a finite perpendicular pressure downstream. This may be interpreted as being due to an increase of the degrees of freedom from 1 to 3 in the gas. In the numerical analysis below, we have checked for the entropy increase as the physicality criterion for the solution.

The easiest way to analyze the compression ratio as a function of Mach number is to solve for $b$ or $\beta_{\|}$in Eqs. (22-23), regard it as a function of two variables $(r$ and $M)$, and to contour plot it fixing the values of other parameters. Thus, e.g.,

$$
\begin{aligned}
\beta_{\|}= & \frac{2(4-r)}{5 r} M^{2}+\frac{4\left[\phi_{2}-1-r\left(\phi_{1}-1\right)\right]}{5(r-1)} \\
& -\frac{b}{5(r-1)}\left\{2 M^{2} r-5+2\left(\phi_{1}-1\right)(2 r-5)\right. \\
& \left.-r\left[2 M^{2}-5 r-6 r\left(\phi_{2}-1\right)\right] \frac{\left(M^{2}-\phi_{1}\right)^{2}}{\left(M^{2}-\phi_{2} r\right)^{2}}\right\},
\end{aligned}
$$

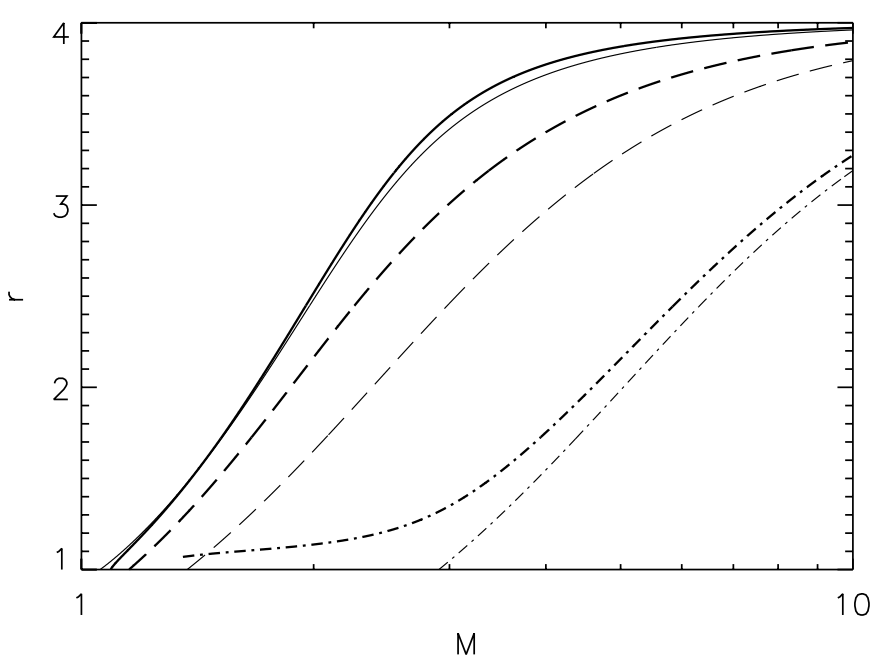

Fig. 1. Gas compression ratio of a parallel shock with $\phi_{2}=1$ propagating into a plasma with $\beta_{\|}=0.2$ (solid curves), 2.0 (dashed curves), and 10.0 (dot-dashed curves) with $b=0.1$, and $\phi_{1}=1$ (thin curves) or $\phi_{1}=\max \left\{0,1-\frac{1}{2} \beta_{\|} /(1+b)\right\}$ (thick curves).

which has been used in Fig. 1 to present the fast shock solution for an isotropic downstream pressure in case of large-amplitude upstream waves $(b=0.1)$. For comparison, both low- and high-beta upstream plasmas are considered with isotropic and very anisotropic conditions. The effect of upstream anisotropy is, as expected, small for shocks with cold upstream gas, but it becomes significant as the beta becomes larger. The effect of anisotropy for a large beta is to increase the compression ratio, i.e., to give a stronger shock for a constant Mach-number. Note that in case of the largest beta, we have had to discard the solutions with smallest values of $r$ as unphysical since they do not fullfil the entropy-increase criterion.

The Alfvén-wave transmission coefficients for the shocks in Fig. 1 are given in Fig. 2. We choose outwardspropagating $(h=-1)$ upstream waves consistent with their generation by counter-streaming particles. For the colder upstream gas, the transmission coefficients are affected only a little by the pressure anisotropy, but for the hotter case we see substantial effects; especially the reflection coefficient is substantially increased in presence of anisotropies. Generally, $T>R$, for $h=-1$, but for a marginally firehose-stable upstream plasma $\left(\phi_{1}=0\right)$, the downstream state tends to an equipartition between forward and backward propagating Alfvén waves $(T, R \rightarrow 2)$ as $r \rightarrow 4$.

\subsection{Test-particle acceleration}

The most important parameter controlling the energy spectrum of cosmic rays accelerated by a shock wave is the compression ratio of the scattering centers that are responsible for the isotropization of the energetic 


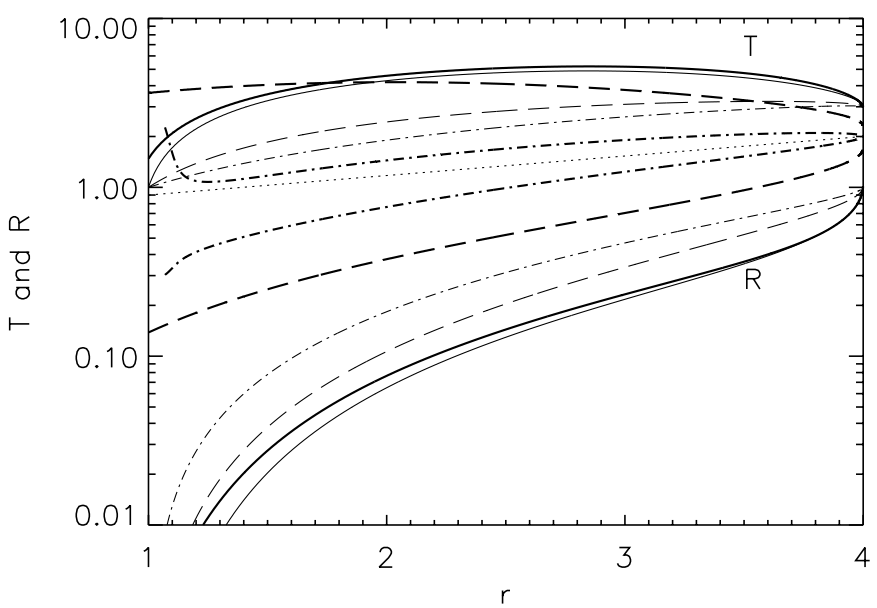

Fig. 2. The transmission coefficients $T$ and $R$ for parallel shocks with $\phi_{2}=1$ propagating into a plasma with $\beta_{\|}=0.2$ (solid curves), 2.0 (dashed curves), and 10.0 (dotdashed curves) with $b=0.1$, and $\phi_{1}=1$ (thin curves) or $\phi_{1}=\max \left\{0,1-\frac{1}{2} \beta_{\|} /(1+b)\right\}$ (thick curves). The dotted line separates the curves for $R$ and $T(>R)$.

particles. In case of Alfvén waves generated in the upstream region, we may write

$r_{k}=\frac{u_{1}-\phi_{1}^{1 / 2} V_{\mathrm{A} 1}}{u_{2}+H_{c 2} \phi_{2}^{1 / 2} V_{\mathrm{A} 2}}$

where

$H_{c 2}=-\frac{T_{k}^{2}-R_{k}^{2}}{T_{k}^{2}+R_{k}^{2}}$

is the downstream cross helicity, and $T_{k}$ and $R_{k}$ are the transmission coefficients of the waves at constant wavenumber $k$. Generally, they depend on the spectral index of the wave intensity, $I(k) \propto k^{-q}$, but for $q=1$ they reduce to $T$ and $R$, respectively (Vainio \& Schlickeiser 1999). Thus, for $q=\phi_{2}=1$,

$r_{k}=r \frac{\left(T^{2}+R^{2}\right)\left(M-\phi_{1}^{1 / 2}\right)}{\left(T^{2}+R^{2}\right) M-\left(T^{2}-R^{2}\right) r^{1 / 2}}$

which is plotted in Fig. 3 for the shocks analyzed in Figs. 1 and 2. The effect of anisotropy is again negligible for the shock propagating into the colder gas, but for the hotter gas, the shock is much stronger particle accelerator in case of anisotropic upstream gas. The shocks with small $\beta_{\perp}$ produce the largest values $(\approx 6.5$ for the studied case of $b=0.1)$ of the scattering-center compression ratio at relatively low $r$ and $M$, which was already the result of Vainio \& Schlickeiser $(1998,1999)$. The inclusion of anisotropic pressure, however, allows an extension of this interesting parameter range, where the spectral index of the shockaccelerated particles is between $1<\Gamma<2$, to shocks propagating into hotter gases than for the isotropic case.

The results for the scattering-center compression ratio were also calculated for the interesting case of $\beta_{\|}=2$ at several values of the firehose factor (Fig. 4) to see, how

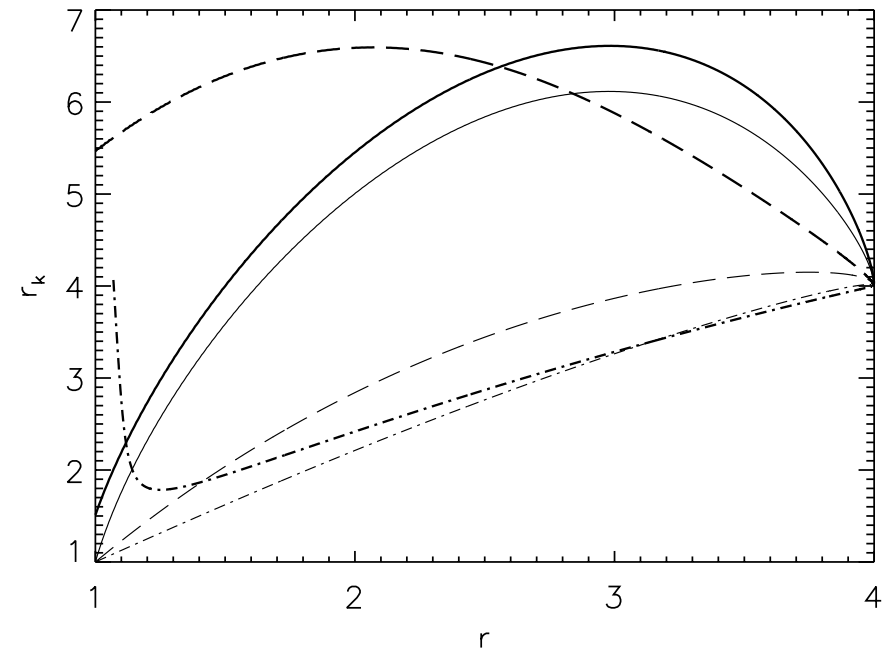

Fig. 3. Scattering-center compression ratio of a parallel shock with $\phi_{2}=1$ propagating into a plasma with $\beta_{\|}=0.2$ ( solid curves), 2.0 (dashed curves), and 10.0 (dot-dashed curves) with $b=0.1$, and $\phi_{1}=1$ (thin curves) or $\phi_{1}=\max \left\{0,1-\frac{1}{2} \beta_{\|} /(1+\right.$ b)\} (thick curves).

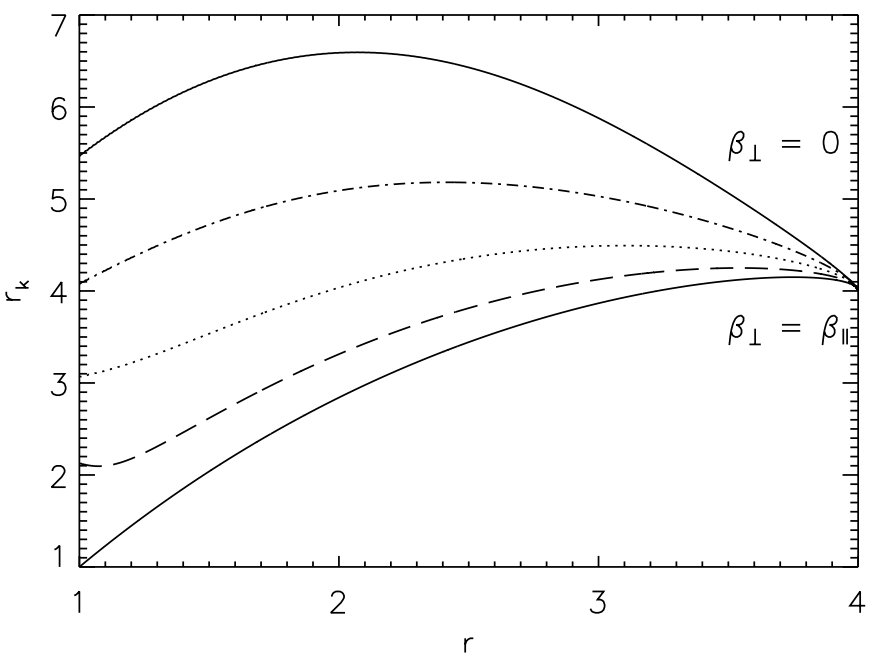

Fig. 4. Scattering-center compression ratio of a parallel shock with $\phi_{2}=1$ propagating into a plasma with $b=0.1$ and $\beta_{\|}=2.0$, with $\beta_{\perp}=0, \beta_{\|}$(solid curves) and $\beta_{\perp} / \beta_{\|}=0.75$ (dashed curve), 0.5 (dotted curve), and 0.25 (dot-dashed curve).

large anisotropies are needed for qualitative effects on particle acceleration from pressure anisotropies. It is evident that relatively small values, below $\phi_{1} \approx 0.5$, are needed to produce large effects on particle acceleration. But, for $\phi_{1}<0.25$ the scattering-center compression ratio is above 4 for all $1<r<4$.

Perhaps the most unexpected result of our calculation is the ability of weakly compressive $(r \gtrsim 1)$ shocks to accelerate particles efficiently in warm plasmas. This result, however, depends crucially on the assumption of the shocks ability to isotropize the fluid. If $r$ differs considerably from unity, one can probably safely assume that the 
small-wavelength magnetic turbulence generated in the shock transition can be responsible for the isotropization. On the other hand, the transverse magnetic field attains large values across the $\beta_{\|} \sim 2$ shocks with $\phi_{1} \ll 1$ regardless of the compression ratio. This can also add to the isotropization of the particles since the field changes direction across the shock at scales comparable to the ion Larmor radius. However, the shocks with small $r$ do not contain large amounts of energy to be given for accelerated particles. When at the same time the test-particle spectral index is below 2, the pressure in accelerated particles diverges without a cutoff in the spectrum. The less there is energy available, the smaller value for the cutoffmomentum must be, and it may well turn out that the weak shocks can not be described by the test-particle picture at all. The confirmation of this requires non-linear analysis, which we shall undertake in the future.

\subsection{Pressure anisotropies created by non-thermal particles}

We will briefly discuss the pressure anisotropies created by the shock-related non-thermal particles assuming nonrelativistic particle speeds for simplicity. For particles accelerated at the shock to speeds clearly exceeding the shock-frame scattering-center speed, $V_{1}$, particle scattering results in a distribution function, $f_{\mathrm{st}}(x, v, \mu)=$ $\mathrm{d}^{6} N /\left(\mathrm{d}^{3} v \mathrm{~d}^{3} x\right)$, that is only weakly dependent on the pitch-angle, $\arccos \mu$. In the upstream wave frame, the small anisotropic portion of the distribution, $g_{\mathrm{st}}(\mu)=$ $f_{\text {st }}-\left\langle f_{\text {st }}\right\rangle$ where $\langle\cdot\rangle$ denotes angle averaging, is antisymmetric relative to $\mu=0$ if the net magnetic helicity of the waves is zero (e.g., Schlickeiser 1989), which we assume. Thus, the pressures due to these particles are

$$
\begin{aligned}
P_{\| \mathrm{st}} & =m \int \mathrm{d}^{3} v v^{2}(\mu-\epsilon)^{2} f_{\mathrm{st}} \\
P_{\perp \mathrm{st}} & =\frac{m}{2} \int \mathrm{d}^{3} v v^{2}\left(1-\mu^{2}\right) f_{\mathrm{st}},
\end{aligned}
$$

where $\epsilon=\left(u_{1}-V_{1}\right) / v=\phi_{1}^{1 / 2} V_{\mathrm{A} 1} / v$ and the integrals are calculated in the wave frame. Using the antisymmetry of $g_{\text {st }}$ and the zero net flux of particles in the shock frame, $\int \mathrm{d}^{3} v\left(v \mu+V_{1}\right) f_{\text {st }}=0$, one obtains after a straight-forward calculation the contribution from these particles to the upstream pressure anisotropy as

$P_{\| \mathrm{st}}-P_{\perp \mathrm{st}}=\phi_{1}^{1 / 2}\left(2 M-\phi_{1}^{1 / 2}\right) \frac{\rho_{\mathrm{st}}(x)}{\rho_{1}} \frac{B_{0}^{2}}{4 \pi}$.

This increases towards the shock with the suprathermal particle density $\rho_{\text {st }}$, but does not attain large values for low-Mach-number shocks. Note that this contribution is exactly zero for a marginally stable plasma $\left(\phi_{1}=0\right)$.

Another contribution to the pressure anisotropy comes from the particles that have just been reflected by the shock and subsequently been picked up by the upstream waves. Let us, for simplicity, consider a cold background plasma, and assume that the shock reflects a small fraction of the incoming background-plasma ions back to the upstream region. We model those particles as a beam with a density of $\rho_{\mathrm{b}}=m n_{\mathrm{b}}$ propagating along the magnetic field away from the shock and include also the particles that return to the shock after their isotropization by the upstream waves. Specifically, in the upstream wave frame we take the distribution function for the reflected and returning particles to be

$f_{\mathrm{rr}}(x, v, \mu)=n_{\mathrm{b}}(x)\left[\delta(\mu+1)+\frac{1}{2} M /\left(M-\phi_{1}^{1 / 2}\right)\right] F(v),(32)$

where $F(v)=\delta\left\{v-\left(u_{1}+V_{1}\right)\right\} / 2 \pi v^{2}$. This gives a zero net flux of particles in the shock frame and a pressure difference of

$P_{\| \mathrm{rr}}-P_{\perp \mathrm{rr}}=M\left[4 M+\frac{1}{2} \phi_{1} /\left(M-\phi_{1}^{1 / 2}\right)\right] \frac{\rho_{\mathrm{b}}(x)}{\rho_{1}} \frac{B_{0}^{2}}{4 \pi}$.

If this contribution dominates the upstream pressure anisotropy, one can obtain a cubic equation for $\phi_{1}^{1 / 2}$. At high Mach numbers, the solution is approximately $\phi_{1}=1-4 M^{2} \rho_{\mathrm{b}} / \rho_{1}$.

\section{Conclusion}

We have considered the Alfvén-wave transmission and test-particle acceleration problem (Vainio \& Schlickeiser 1998, 1999) for shocks with anisotropic pressure. In our model, the firehose factors on both sides of the shock are predetermined parameters. For a detailed analysis, we chose a model with isotropic downstream pressure, and upstream pressure anisotropy that is bounded by the requirement of firehose stability. We showed that the pressure anisotropies have only a minor effect on wave transmission and particle acceleration for plasmas with low $\beta$. However, plasmas with upstream $\beta_{\|} \sim 2$ and $\beta_{\perp} \ll \beta_{\|}$seem to develop qualitative effects on both the wave transmission and particle acceleration relative to the isotropic-pressure case. Our study revealed the capability of weak shocks propagating into such plasmas to accelerate particles effectively by creating a large change in the average scattering-center speed across the shock though the increase of the phase speed of low-frequency waves across the shock. Plasmas with large values of beta, $\beta_{\|} \gg 1$, can not develop very large anisotropies $\left(\beta_{\perp} \ll \beta_{\|}\right)$without becoming firehose unstable, which again prevents large deviations in our model between the anisotropic and isotropic cases except for the weakest shocks with $M<M_{\text {isotr }}(r=1)$. The low-Mach-number shocks are weakly entropy increasing in the ideal gas model and should, therefore, be studied more carefully using kinetic analysis. In conclusion, the results of our study point out the importance of kinetic analysis of upand downstream plasma to fully understand the physics of shock acceleration.

Acknowledgements. R. V. acknowledges the financial support of the Academy of Finland (project \# 46331) and the PLATON Network (EC contract \# HPRN-CT-2000-00153). 


\section{References}

Axford, W. I., Leer, E., \& Skadron, G. 1977, Proc. 15th Int. Cosmic-Ray Conf., 11, 132

Bell, A. R. 1978, MNRAS, 182, 147

Blandford, R. D., \& Ostriker, J. P. 1978, ApJ, 221, L29

Blandford, R. D., \& Eichler, D. 1987, Phys. Rep., 154, 1

Boyd, T. J. M., \& Sanderson, J. J. 1969, Plasma Dynamics (Thomas Nelson and Sons Ltd., London)

Drury, L. O' C. 1983, Rep. Progr. Phys., 46, 973

Krymsky, G. F. 1977, Dokl. Akad. Nauk. SSSR, 243, 1306

Lyu, L. H., \& Kan, J. R. 1986, J. Geophys. Res., 91, 6771
Marsch, E. 1991, in Physics of the Inner Heliosphere, vol. II, ed. R. Schwenn, \& E. Marsch (Springer-Verlag, Berlin Heidelberg), 45

McKenzie, J. F., \& Westphal, K. O. 1969, Planet. Space Sci., 17,1029

Schlickeiser, R. 1989, ApJ, 336, 234

Schlickeiser, R., \& Vainio, R. 1998, Ap\&SS, 264, 457

Stix, T. H. 1962, The Theory of Plasma Waves (McGraw-Hill Book Company Inc., New York)

Vainio, R., \& Schlickeiser, R. 1998, A\&A, 331, 793

Vainio, R., \& Schlickeiser, R. 1999, A\&A, 343, 303 